\title{
ENDOVASCULAR THERAPY WITH OR WITHOUT INTRAVENOUS LOW DOSE ALTEPLASE IN ACUTE STROKE PATIENTS WITH ANTERIOR LARGE VESSEL OCCLUSION
}

Hiroshi Yamagami ${ }^{1}$, Kazutaka Uchida ${ }^{2}$, Shinichi Yoshimura ${ }^{2}$, Takeshi Morimoto ${ }^{3}$, Nobuyuki Sakai ${ }^{4}$, Kazunori Toyoda ${ }^{5}$. and RESCUE-Japan Registry 2 Investigators

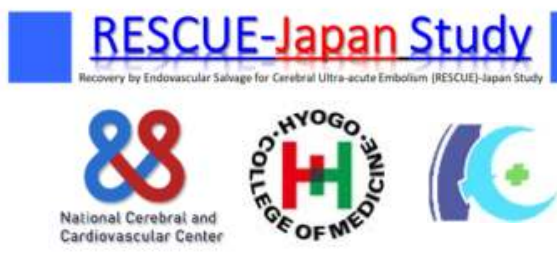

1. Div. of Stroke Care Unit, National Cerebral and Cardiovascular Center, Suita, JAPAN

2. Dept. of Neurosurgery, Hyogo College of Medicine, Nishinomiya, JAPAN

3. Dept of Clinical epidemiology, Hyogo College of Medicine, Nishinomiya, JAPAN

4. Dept of Neurosurgery, Kobe City Medical Center General Hospital, Kobe, JAPAN

5. National Cerebral and Cardiovascular Center, Suita, JAPAN

Table 1. Patient Characteristics

\section{Background and Purpose}

$>$ Mechanical thrombectomy is now recommended as essential treatment for patients with acute ischemic stroke due to anterior large vessel occlusion.

> However, whether pre-treatment with intravenous thrombolysis (IVT) provides any additional benefits to the patients undergoing mechanical thrombectomy remains unclear.

> IVT with low dose $(0.6 \mathrm{mg} / \mathrm{kg})$ alteplase can reduce symptomatic intracranial hemorrhage $(\mathrm{sICH})$ compared with standard dose. In Japan, low dose alteplase is approved for patients with acute ischemic stroke within $4.5 \mathrm{~h}$ from onset.

$>$ We aimed to clarify the impact of prior IVT using low dose alteplase on outcomes in large vessel acute stroke patients undergoing endovascular therapy.

\section{Methods}

In a Japanese nationwide registry study, RESCUE-Japan Registry 2 (Yoshimura S, et al. J Am Heart Assoc. 2018), 2420 patients with acute stroke due to large vessel occlusion were registered between October 2014 and January 2017. Among them, 595 patients with occlusion of the internal carotid artery or the middle cerebral artery M1 portion and who underwent endovascular therapy within 6 hours after onset were analyzed.

Functional outcome assessed by modified Rankin Scale (mRS) score at 90 days and incidence of $\mathrm{sICH}$ (with increasing NIHSS score $\geqq 4$ ) within 72 hours were compared between patients with and without prior IVT.

\section{Results}

Figure 1. $\mathrm{mRS}$ at 90 days by prior IVT

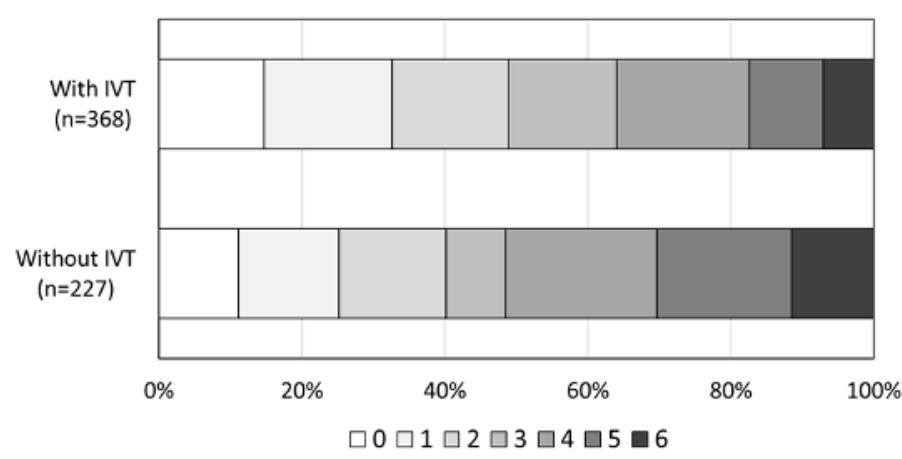

Common OR $1.67(95 \% \mathrm{Cl} 1.25-2.24, \mathrm{P}=0.001)$

Adjusted* common OR 1.38 (95\% Cl 0.99-1.91, P=0.06)

*Adjusted factors: age, sex, SBP, DBP, Af, previous stroke, baseline NIHSS, ASPECTS, Glucose, Creatinine, ICA occlusion, prior anticoagulants, onset to puncture time, TICI 2b-3

\begin{tabular}{|c|c|c|c|}
\hline & $\begin{array}{c}\text { With IVT } \\
n=368\end{array}$ & $\begin{array}{l}\text { Without IVT } \\
n=227\end{array}$ & $P$ \\
\hline \multicolumn{4}{|l|}{ Demographics } \\
\hline Mean age, years (SD) & $73.8 \pm 11.5$ & $73.4 \pm 12.6$ & 0.70 \\
\hline Men, $\mathrm{n}(\%)$ & $234(64)$ & $134(60)$ & 0.34 \\
\hline \multicolumn{4}{|l|}{ Past medical history } \\
\hline Hypertension, $\mathrm{n}(\%)$ & $203(55)$ & $125(55)$ & 1.00 \\
\hline Diabetes mellitus, $\mathrm{n}(\%)$ & $65(18)$ & $41(18)$ & 0.91 \\
\hline Dyslipidemia, $\mathrm{n}(\%)$ & $82(22)$ & $41(18)$ & 0.25 \\
\hline Atrial fibrillation, $\mathrm{n}(\%)$ & $174(47)$ & $125(55)$ & 0.08 \\
\hline Current Smoking, n (\%) & $56(15)$ & $33(15)$ & 0.91 \\
\hline Previous stroke, $\mathrm{n}(\%)$ & $13(4)$ & $22(10)$ & $<0.01$ \\
\hline \multicolumn{4}{|l|}{ Baseline characteristics } \\
\hline NIHSS score & $17(14-22)$ & $18(14-23)$ & 0.08 \\
\hline Systolic blood pressure, $\mathrm{mmHg}$ & $155 \pm 26$ & $152 \pm 27$ & 0.25 \\
\hline Diastolic blood pressure, $\mathrm{mmHg}$ & $85 \pm 18$ & $84 \pm 18$ & 0.36 \\
\hline Hemoglobin, $\mathrm{g} / \mathrm{dL}$ & $13.4 \pm 1.9$ & $12.8 \pm 2.1$ & $<0.01$ \\
\hline Creatinine, $\mathrm{mg} / \mathrm{dL}$ & $0.94 \pm 0.70$ & $1.14 \pm 1.66$ & 0.04 \\
\hline Glucose, $\mathrm{mg} / \mathrm{dL}$ & $140 \pm 46$ & $140 \pm 52$ & 0.96 \\
\hline Prior antiplatelet therapy, $\mathrm{n}(\%)$ & $69(19)$ & $54(24)$ & 0.12 \\
\hline Prior anticoagulant therapy, $\mathrm{n}(\%)$ & $60(16)$ & $80(36)$ & $<0.01$ \\
\hline \multicolumn{4}{|l|}{ Imaging Characteristics } \\
\hline Baseline ASPECTS* & $8(6-9)$ & $7(5-9)$ & $<0.01$ \\
\hline ICA occlusion, $\mathrm{n}(\%)$ & $175(48)$ & $109(48)$ & 0.93 \\
\hline \multicolumn{4}{|l|}{ Treatment process times } \\
\hline Onset to Door, $\min$ & $75(45-135)$ & $97.5(45-184)$ & 0.02 \\
\hline Onset to IV rt-PA, min & $120(90-160)$ & - & - \\
\hline Onset to groin puncture, $\min$ & $170(120-215)$ & $180(130-270)$ & $<0.01$ \\
\hline Onset to reperfusion, $\min$ & $228(170-290)$ & $258(185-335)$ & $<0.01$ \\
\hline \multicolumn{4}{|l|}{ Reperfusion after EVT } \\
\hline $\mathrm{TICl} 2 \mathrm{~b}-3, \mathrm{n}(\%)$ & $326(89)$ & $196(87)$ & 0.52 \\
\hline
\end{tabular}

Table 2. Impact of prior IVT on outcomes

\begin{tabular}{|l|c|c|c|c|}
\hline & $\begin{array}{c}\text { With IVT } \\
\mathrm{n}=368\end{array}$ & $\begin{array}{c}\text { Without IVT } \\
\mathrm{n}=227\end{array}$ & $\begin{array}{c}\text { OR } \\
(95 \% \mathrm{Cl})\end{array}$ & $\begin{array}{c}\text { Adjusted OR* } \\
(95 \% \mathrm{Cl})\end{array}$ \\
\hline $\begin{array}{l}\text { mRS score 0-3 } \\
\text { at 90 days }\end{array}$ & $\begin{array}{c}236 \\
(64.1 \%)\end{array}$ & $\begin{array}{c}110 \\
(48.5 \%)\end{array}$ & $\begin{array}{c}1.90 \\
(1.36-2.66) \\
\mathrm{P}<0.01\end{array}$ & $\begin{array}{c}1.63 \\
(1.05-2.53) \\
\mathrm{P}=0.03\end{array}$ \\
\hline $\begin{array}{l}\text { mRS score 0-2 } \\
\text { at 90 days }\end{array}$ & $\begin{array}{c}180 \\
(48.9 \%)\end{array}$ & $\begin{array}{c}91 \\
(40.1 \%)\end{array}$ & $\begin{array}{c}1.43 \\
(1.02-2.00) \\
\mathrm{P}=0.04\end{array}$ & $\begin{array}{c}1.21 \\
(0.78-1.88) \\
\mathrm{P}=0.39\end{array}$ \\
\hline $\begin{array}{l}\text { Any ICH within } \\
72 \text { hours } \\
\text { (n=554) }\end{array}$ & $\begin{array}{c}94 / 348 \\
(27.0 \%)\end{array}$ & $\begin{array}{c}5 / 206 \\
(27.2 \%)\end{array}$ & $\begin{array}{c}0.99 \\
(0.67-1.46) \\
\mathrm{P}=0.97\end{array}$ & $\begin{array}{c}1.45 \\
0.92-2.27) \\
\mathrm{P}=0.11\end{array}$ \\
\hline $\begin{array}{l}\text { sICH within } \\
72 \text { hours } \\
\text { (n=554) }\end{array}$ & $\begin{array}{c}10 / 348 \\
(2.9 \%)\end{array}$ & $\begin{array}{c}6 / 206 \\
(2.9 \%)\end{array}$ & $\begin{array}{c}0.99 \\
(0.35-2.75) \\
\mathrm{P}=0.98\end{array}$ & $\begin{array}{c}1.07 \\
0.35-3.36) \\
\mathrm{P}=0.91\end{array}$ \\
\hline
\end{tabular}

*Adjusted factors: age, sex, SBP, DBP, Af, previous stroke, baseline NIHSS, ASPECTS,

Glucose, Creatinine, ICA occlusion, prior anticoagulants, onset to puncture time, TICI 2b-3

\section{Conclusion}

Prior IVT with low dose alteplase before endovascular therapy can modestly improve functional outcome without increasing risk of $\mathrm{sICH}$ in acute stroke patients. 